gerührt und innig gemischt werden. Den Koksverbrauch beim Brennen der Zementklinker, der ursprünglich $70 \mathrm{~kg}$ auf das $\mathrm{Faß}$ Zement betrug, verringerte er, und an Stelle der ursprünglich fast ausschlieBlichen Handarbeit wandte er immer mehr Maschinenarbeit an. Wenn auch das Vorurteil der Kundschaft gegenüber dem de u t s chen Erzeugnis erst gebrochen werden mußte, und wenn die unerwarteten Schwierigkeiten, die die bisher unerklärt gebliebene Umwandlung des früher schnell abbindenden Zementes in einen „Langsambinder" machte, erst überwunden werden mußte,so blühte doch das Unternehmen in den 60er Jahren mächtig auf und gab Veranlassung dazu, da $\int$ an vielenOrten in Deutschland und auf dem Kontinent Zementfabriken nach Stettiner Muster entstanden.

Die älteste Portlandzementfabrik ist aber nicht müßig geblieben, sondern ist in jeder Beziehung bestrebt gewesen, die eigenen Erfahrungen und die verwandter Industrien in ihrem Betriebe zu verwerten. Die Zementziegel wurden nicht mehr mit der Hand gestrichen, sondern mit Ziegelpressen; zum Trocknen der Ziegel wurde die Abhitze der Öfen verwendet. Im Jahre 1868 wurden von $\mathrm{H}$ of $\mathrm{f} \mathrm{m}$ a $\mathrm{n} n$ die ersten Ringöfen für Zement an Stelle der alten Schachtöfen in Züllchow gebaut und nach Überwindung vieler Schwierigkeiten in Gang gesetzt. Die Mühlenarbeit wurde erleichtert durch die Einführung von Steinbrechern, und in den 90er Jahren wurden schließlich die Mahlgänge vollständig ausgeschaltet und durch Griffinmühlen ersetzt. Auch die Ringöfen wurden in den $80 \mathrm{er}$.Jahren des vergangenen Jahrhunderts verdrängt durch die Etagenöfen von $\mathrm{D}$ i et z s c h, welche sich für die Erzeugung der hohen Zementbrenntemperatur geeigneter erwiesen. Vorbildlich ist die Züllchower Fabrik gewesen durch Beseitigung des Staubes in der Mühle und im Packraum. Der feine Zementstaub war in den ersten Jahrzehnten der Fabrikation ein Grund zu dauernder Belästigung und Schädigung der Arbeiter und zur Abnutzung der Maschinenlager. Durch geschickt angelegte Saugvorrichtungen gelang es, nicht nur in den Mühlen, sondern, was noch erheblich schwieriger war, bei dem Füllen der Fässer den Staub vollständig abzufangen, anzusammeln und als wertvolles Material dem Betriebe wieder zuzuführen. In großherziger Weise verzichtete die Fabrik auf die Patentierung der hierzu ersonnenen Vorrichtungen. Sie machto vielmehr ihre Erfindung zugunsten der Arbeiter in den Zementfabriken der gesamten Konkurrenz zugänglich. Ein besonderes Verdienst Dr. De J brücks war es, da $\beta$ er durch den von ihm ins Leben gerufenen $\mathrm{V}$ erein $\mathrm{Deuts}$ cher Portlan dzementfabrikanten und die von diesem Verein geschaffenen Normen für eine gleichmäßige gute Qualität des deutschen Portlandzementes sorgte; auch die Erfindung der ersten für die Untersuchung von Portlandzement geeigneten Vorrichtungen und Apparate ist $\mathrm{D}$ e $\mathrm{l} \mathrm{br}$ ü c k s Verdienst.

Die beiden Männer, welche in jahrzehntelanger Arbeit ihre eigene Fabrik und die gesamte deutsche Industrie so mächtig gefördert haben, weilen nicht mehr unter den Lebenden. Lossius ist im Jahre 1886 durch J on a s, und dieser im Jahre 1905 durch $\mathrm{H}$. Kirs ch ersetzt worden. An Stelle von D e l b r $\ddot{\mathrm{u}} \mathrm{c} \mathbf{k}$ trat im Jahre 1893 Dr. K. G o s l i c h, der in der Fabrik seit dem Jahre 1876 als Betriebschemiker wirkte. Der alte Geist aber ist trotz aller durch die Überproduktion in Zement erwachsenen Schwierigkeiten in der Züllchower Fabrik geblieben, ein Geist, der sich auch dadurch kund gibt, daß in den 50 Jahren des Bestehens der Fabrik nur einmal ein partieller Streik, und auch dieser nur wegen gekränkten Ehrgefühles der zunftmäßigen Böttcher, vorgekommen ist. Seit dem Jahre 1890 hat die Fabrik einen ArbeiterausschuB, mit welchem die Direktion alle wichtigeren Angelegenheiten, die die Arbeiter irgendwie betreffen, berät und beschließt. Gegenüber den Versuchen der neueren Zeit, unter dem Namen Portlandzement andere Produkte in den Handel zu bringen, hält die Fabrikleitung unerschütterlich fest an den letzten Worten, die Dr. $\mathrm{D}$ e l b r ü ck zu dem jetzigen technischen Direktor gesprochen hat: „L a s s e n $\mathrm{S}$ i e sich nicht irre machen und halten Siemir unsere Qualität hoc $h$ ".

R.

\section{Umwandlung der Dextrose in Lävulose u. Nachweis der Lävulose. ${ }^{1}$ ) \\ Von H. Osm, Hannover. \\ (Eingeg. d. 29./6. 1905.)}

Wenn man Stärke mit Malz verzuckert und die entstandenen Maltosesirupe mit starkem Alkohol auszieht, so findet man in den leicht löslichen Fraktionen regelmäßig Dextrose und Lävulose; letztere verrät sich durch ihr niedriges Drehungsvermögen und durch die Bräunung und Säuerung beim Eindampfen. Diese Lävulose kann, wie die Dextrose, aus dem Malze stammen, denn

1) Vortrag, gehalten auf der Hauptversammlung des V. d. Ch. zu Bremen am 17./6. 1905. 
im Malze sind beide Monosaccharide und Saccharose wiederholt nachgewiesen worden; nach Brown und Morris vermag der wachsende Keimling Maltose in Saccharose umzusetzen $^{2}$ ).

Dieselben niedrig polarisierenden Monosaccharide können auch bei der Verzuckerung der Stärke mit Säuren entstehen.

Dierssen ${ }^{3}$ ) isolierte bei seiner Stärkehydrolyse durch Oxalsäure Produkte von $[\alpha]_{\mathrm{D}}+23^{\circ}$, mit einem Reduktionsvermögen von $86 \%$ (Dextrose $=100)$; nach weiterer Abscheidung kristallisierender Dextrose ging die Drehung der in Alkoholäther löslichen Anteile bis $[\alpha]_{\mathrm{D}}+4^{\circ}$ herunter. $\mathrm{D}$ i e r s s e $\mathrm{n}$ hält sein Produkt für ein Gemenge von Dextrose und Lävulose und meint, daß Lävulose möglicherweise ein normales $\mathrm{Ab}$ bauprodukt der Stärke sei. Die nähere Untersuchung hat gezeigt, daß hier in der Tat Lävulose vorliegt, daß sie aber nicht primär aus Stärke, sondern sekundär durch Einwirkung der Säure auf Dextrose entsteht. Es war das nicht zu erwarten, da Lävulose gegen Säuren, wenigstens gegen warme, sehr unbeständig ist und im käuflichen Stärkezucker nicht vorzukommen scheint.

Reichlich erhielt ich Lävulose aus Dextrose durch längere Einwirkung starker kalter Schwefelsäure. $1,1 \mathrm{~kg}$ kristallisierte (= $1 \mathrm{~kg}$ wasserfreie) Dextrose wurde in einem Gemisch von 21 Wasser und 11 konz. Schwefelsäure kalt gelöst und 4 Monate bei Zimmertemperatur hingestellt; alsdann wurde die braune Flüssigkeit mit reinem Calciumcarbonat neutralisiert, filtriert und bei mäßiger Temperatur eingedampft. Unterwirft man diese Lösung einer Gärung mit Bierhefe, so bleibt von Zuckerarten nur die unvergärbare Isomaltose E. F is chers übrig, die, wie ich früher gezeigt habe, auf diese Weise in beträchtlicher Menge, leider nur in Sirupform, zu erhalten ist ${ }^{4}$ ). Unterbleibt aber die Gärung, und wird die Lösung vorsichtig zum dicken Sirup eingeengt, so kristallisiert zunächst sehr viel unveränderte Dextrose aus, und die davon getrennten Muttersirupe besitzen eine weit unter $[\alpha]_{\mathrm{D}}+50^{\circ}$ liegendes Drehungsvermögen. Die Zergliederung der Produkte geschieht, wie früher beschrieben ${ }^{5}$ ), durch fraktioniertes Lösen in 95- bis $100 \%$ igem Äthylalkohol, verbunden mit Kristallisation; auch hier lassen sich die Dextrosekristalle durch Waschen mit ab-

2) Moritz und Morris. Windisch, Handbuch der Brauwissensehaft 1893, 68 .

3) Diese Z. 1903, 128; 1904, 1174. - Vgl. auch Rosin, Chem.-Ztg. 1903, Ref. 172. - Erlen. $\mathrm{m}$ e y e r, J. prakt. Chem. 1905, 71, 382.

4) Chem.-Ztg. 1896, 761 .

5) Diese Z. 1904, 1663. solutem Äthylalkohol glatt von den dicken Muttersirupen trennen, so daß die Trennung selbst bei kleineren Mengen ausführbar ist. Nachdem die Hauptmenge der Dextrose entfernt worden ist, wollen die Muttersirupe bald nicht mehr kristallisieren; man erzwingt aber durch wiederholtes Zerlegen mit Äthylalkohol weitere Kristallisationen; am leichtesten kristallisieren die Auszüge vom niedrigsten Drehungsvermögen, von $0^{\circ}$ bis $[a]_{D}+20^{\circ}$, während diejenigen von über $[\alpha]_{D}+30^{\circ}$ nur sehr langsam oder nicht mehr kristallisieren. Die Kristalle bestanden immer aus Dextrosehydrat, beim öfteren Waschen mit Methylalkohol gingen sie in voluminöses Dextroseanhydrid über. Schließlich resultierten, durch Ausziehen mit Alkoholäther, lävulosereiche Sirupe von $[\alpha]_{\mathrm{D}}-10^{\circ}$ und darunter. Schätzungsweise mögen aus $100 \mathrm{~g}$ Dextrose $20 \%$ Isomaltose und $5 \mathrm{bis}$ $10 \%$ Lävulose entstanden sein; ca. $60 \%$ wurden als kristallisierte Dextrose zurückgewonnen.

Der exakte $\mathrm{Nachweis} \mathrm{der} \mathrm{L} \mathrm{ä-}$ $\mathrm{vulose}$ in unbekannten Gemischen ist nicht ganz einfach. Von den 4 bekannten vergärbaren Monosacchariden kristallisieren Galaktose und Dextrose auch aus unreinen Sirupen leicht; noch leichter ist Mannose durch ihr schwer lösliches Phenylhydrazon zu erkennen und zu isolieren; die Lävulose dagegen kristallisiert niemals aus unreinen Sirupen, sie bildet keine isolierbaren Hydrazone, und das Osazon teilt sie mit Dextrose und Mannose. Die von S e I iw a n of $f$ vorgeschlagene Reaktion, Rotfärbung mit Resorcin und Salzsäure, kommt allen Ketosen zu und kann auch bei reiner Dextrose zu Täuschungen führen. Etwas sicherer ist der Nachweis der Lävulose, insonderheit neben Dextrose, nach $\mathrm{Si}$ e b e $\mathbf{n}^{6}$ ). Erwärmt man ein Gemisch von gleichen Mengen reiner Dextrose und Lävulose in nicht mehr als $1 \%$ iger Lösung, mit Salzsäure von $71 / 2 \%$ Gehalt, 3 Stunden im siedenden Wasserbade, so ist die Lävulose unter Braunfärbung und Abscheidung brauner Flocken zerstört, und man findet mit Feh ling scher Lösung fast genau die Dextrose wieder. Aber das Ergebnis wechselt mit dem Mengenverhältnis von Lävulose zu Dextrose; natürlich, denn das Reduktionsvermögen der Lävulose wird nicht völlig vernichtet, und andererseits wird auch die Dextrose von der Salzsäure etwas angegriffen. Die S i e b e n sche Reaktion kann bei der Analyse von Invertzucker, Honig und Fruchtsäften, in Verbindung mit den üblichen Zuckerbe-

$\left.{ }^{6}\right)$ von Lippmann, Chemie der Zuckerarten 1904,895 . 
stimmungsmethoden, gute Dienste leisten, ist aber bei unbekannten Gemengen nur mit Vorsicht zu verwenden.

Zum sicheren Nachweis der Lävulose ist ihre Abscheidung als $\mathrm{Calciumlävu}$ los at nach Dubrunfaut ${ }^{7}$ ) erforderlich; mit 5-10 g eines an Lävulose angereicherten Sirups kommt man damit sicher zum Ziele. Man kühlt die etwa $10 \%$ ige Zuckerlösung in einer Kältemischung bis zum teilweisen Gefrieren $a b$ und mischt auf 10 Teile Gesamtzucker etwa 6 Teile trockenes gesiebtes Kalkhydrat hinzu, unter kräftigem Durchschütteln. Eine normale Invertzuckerlösung erstarrt dann in wenigen Augenblicken zu einer steifen Masse feinster Kristallnädelchen des Monocalciumlävulosats; ein Überschuß an $\mathrm{Ca}(\mathrm{OH})_{2}$ ist nicht nachteilig, es erscheint unter dem Mikroskop in rundlichen Körnchen neben den schleimigen Nadelmassen. Meine Sirupe von $[\alpha]_{D} 0^{\circ}$ gaben noch starke, wenn auch weniger dicke Niederschläge. Es empfiehlt sich nicht, die Zuckerlösung stärker zu verdünnen, um vor der Abscheidung des Lävulosats die Lösung vom überschüssigen $\mathrm{Ca}(\mathrm{OH})_{2}$ abfiltrieren zu können; die Ausbeuten an Lävulosat werden dadurch erheblich schlechter. Nach $1 /{ }_{4}$ Stunde wird abgesaugt, was leicht von statten geht, mit Eiswasser gewaschen, und der Niederschlag unter Zusatz von Eisstückchen durch Oxalsäure zerlegt, bis nach wiederholtem Durchschütteln eine schwach saure Reaktion stehen bleibt. Die Filtrate werden bei $50-60^{\circ}$ abgedampft, wobei keinerlei Zersetzung eintritt, wenn die Reaktion, eventuell durch Zutröpfeln von Kalkwasser oder Oxalsäure, eben sauer gehalten wird. $20 \mathrm{~g}$ eines käuflichen Invertzuckers von Gebr. Langelütje (Meißen), mit $[\alpha]_{\mathrm{D}}-15^{\circ}$ und $4 \%$ Rohrzuckergehalt, lieferten auf diese Weise $8 \mathrm{~g}$ Rohlävulose von $[\alpha]_{\mathrm{D}}-73^{\circ}$, also $40 \%$ Ausbeute; diese Rohlävulose zum Kristallisieren zu bringen, gelingt nicht immer; am ehesten erhält man schöne harte Prismen, wenn man den eingedickten Sirup in wenig absolutem Methylalkohol löst, mit Äther bis zur beginnenden Trübung versetzt und geimpft stehen läßt; der Sirup allein kristallisiert in der Regel nicht; aus absolutem Äthylalkohol erhält man zunächst kugelige Warzen. Reine Lävulose besitzt in $10 \%$ iger Lösung bei $+20^{\circ}$ das spezifische Drehungsvermögen von etwa $\left.[\alpha]_{\mathrm{D}}-93^{\circ}\right)$. - Ein anderer käuflicher

7) von Lip pmann, S. 881 .

8) In der neuesten Auflage der Organischen Chemie von $R$ i ch ter-A n s ch ü t z 1903, 659 findet sich die schon vor 10 Jahren berichtigte Zahl $[a] D-71,4^{\circ}$.
Invertzucker (von Hattersheim) mit $[\alpha]_{\mathrm{D}}$ $+10,3^{\circ}$ und $35 \%$ Rohrzuckergehalt, gab aus dem Calciumlävulosat eine Rohlävulose von nur $[\alpha]_{\mathrm{D}}-40$ bis $-50^{\circ}$, sie enthielt vermutlich etwas mitgefällte Saccharose; ein alter Naturhonig lieferte eine Rohlävulose von $[\alpha]_{\mathrm{D}}-60^{\circ}$.

Von meinen aus Dextrose erhaltenen Produkten wurden zwei auf Calciumlävulosat verarbeitet.

A) Sirup $P_{4}, 5$, von $[\alpha]_{\mathbf{D}}-0,6^{\circ}$, von $94 \%$ Reduktionsvermögen (Invertzucker $=$ 100), mit Phenylhydrazin $110 \%$ Phenylosazon liefernd. $\quad 17,7 \mathrm{~g}$ Substanz gaben $3,3 \mathrm{~g}$ Rohlävulose von $[\alpha]_{\mathrm{D}}-66^{\circ}$; also $19 \%$ Ausbeute.

B) Sirup $R_{2,3}$, von $[\alpha]_{D}-6^{\circ}$, von $87 \%$ Reduktionsvermögen und $40,3 \%$ Lävulosegehalt nach S i e b e n. 19,2 g Substanz gaben $5,7 \mathrm{~g}$ Rohlävulose von $[\alpha]_{\mathrm{D}}-63^{\circ}$; also $30 \%$ Ausbeute.

Beide Rohlävulosen wurden vereinigt zum Sirup eingedampft und mit wenig Methylalkohol vermischt; auch hier gelang es mit einiger Geduld, Kristalle zu erzielen.

Die Trennung der Lävulose und Dextrose mittels der Kalksalze bei $0^{\circ}$ ist keine vollständige; während Lävulose in leidlich reinem Zustande isoliert wird, enthalten die löslichen Kalksalze neben Dextrose noch viel Lävulose, auch Spuren von Mannose und andere Produkte. Aus $20 \mathrm{~g}$ normalem Invertzucker von $[\alpha]_{\mathrm{D}}-15^{\circ}$ (s. oben) wurde aus den löslichen Kalksalzen mittels Oxalsäure ein Zuckergemisch von $[\alpha]_{\mathrm{D}}+20,7^{\circ}$ abgeschieden $(12,4 \mathrm{~g})$, daraus wurden gewonnen Dextrosekristalle $(4,2 \mathrm{~g})$ und Restsirupe von $[\alpha]_{\mathrm{D}}+1,8^{0}$ mit einem Reduktionsvermögen von $80 \%$ und einem Lävulosegehalt nach $\mathrm{S}$ i e b e $\mathrm{n}$ von $28 \%$. Ein ganz ähnliches Gemenge gaben die löslichen Kalksalze meiner obigen Sirupe $P_{4,5}$ und $R_{2,3}$, so daß ich aus allen diesen Untersuchungen den Schlu $\beta$ ziehen darf, daß diese, durch Säurewirkung aus Dextrose hervorgegangenen Produkte im wesentlichen aus Lävulose und Dextrose bestehen.

In jüngster Zeit hat $\mathrm{Ne} \mathrm{u} \mathrm{be} \mathrm{r} \mathrm{g} \mathrm{als}$ empfindlichstes Reagens auf Lävulose Methylphenylhydrazin empfohlen ${ }^{9}$ ), insonderheit zu deren Nachweis neben Dextrose im Blutserum und anderen tierischen Säften. Nach $\mathrm{N}$ e u b e r g soll Methylphenylhydrazin $\mathrm{C}_{6} \mathrm{H}_{5}\left(\mathrm{CH}_{3}\right) \mathrm{N}$. $\mathrm{NH}_{2}$, wie alle sekundären unsymmetrischen Hydrazine, nur mit den Ketosen Osazone bilden, nicht aber mit den Aldosen. Ofner ${ }^{10}$ ) hat diese Angabe nicht bestätigt gefunden, vielmehr auch aus Dex-

9) Berl. Berichte 1902, 960.

10) Berl. Berichte 1904, 2623, 3362, 4399, 4616. 
trose dasselbe Methylphenylosazon erhalten, wie aus Lävulose, wenn auch schwieriger; worauf Neuberg dies nur teilweise zugibt und daran festhält, daß Methylphenylhydrazin bei der Prüfung auf Lävulose unter seinen Versuchsbedingungen,,absolut eindeutige "Resultate gebe.

Meine Versuche bestätigen den Befund Ofners, was auch nach der Analogie mit dem Phenylglykosazon plausibler ist. Phenylhydrazin reagiert bekanntlich sowohl mit Dextrose wie mit Lävulose in der Weise, daß beide Hexosen durch überschüssiges Hydrazin zunächst zu ein und demselben Glukoson, $\mathrm{C}_{6} \mathrm{H}_{10} \mathrm{O}_{6}$, oxydiert werden, welches dann mit 2 weiteren Mol. Phenylhydrazin Glukosazon bildet; ebenso wirkt Methylphenylhydrazin. Beide Hydrazinbasen reagieren mit Lävulose schneller und glatter als mit Dextrose, nur ist bei Methylphenylhydrazin der Unterschied viel stärker ausgeprägt, offenbar weil die sekundäre Base die Dextrose schwerer zu Oson oxydiert als die primäre. Aus dem Glukoson und aus Lävulose erhielt E. Fi s e h e r das Methylphenylglukosazon sehr leicht ${ }^{11}$ ); er gab ihm die Formel

$$
\begin{aligned}
\mathrm{CH}_{2} \mathrm{OH}-(\mathrm{CH} . \mathrm{OH})_{3}-\mathrm{C}-\mathrm{CH} \\
\qquad \mathrm{C}_{6} \mathrm{H}_{5}\left(\mathrm{CH}_{3}\right) \mathrm{N}_{2} \quad \mathrm{~N}_{2}\left(\mathrm{CH}_{3}\right) \mathrm{C}_{6} \mathrm{H}_{5}
\end{aligned}
$$

Methylphenylglukosazon entsteht am glattesten in der Kälte. Vermischt man $1 \mathrm{~g}$ reine Lävulose, in $10 \mathrm{~g}$ Wasser gelöst, mit 2,2 ccm reinem Methylphenylhydrazin von Kahlbaum ( $=3^{1} / 2$ Mol.) und $1,2 \mathrm{ccm}$ Eisessig, so löst sich alles klar auf; nach wenigen Stunden beginnen bei Zimmertemperatur schöne gelbe Nadeln auszukristallisieren, die nach 15-20 Stunden die ganze Flüssigkeit zu einem Kristallbrei erstarren machen. Verfährt man in gleicher Weise mit reiner Dextrose, so erscheinen die ersten Nadeln nicht vor 24 bzw. 48 Stunden, auch nicht früher, wenn geimpft und geschüttelt wird; nach 4-5 Tagen ist ebenfalls eine reiche Ausscheidung von gelben Nadeln, aber vermischt mit harzigen Produkten, eingetreten. Wäscht man die Kristalle mit etwas Äther oder kaltem Benzol, worin sich das Harz sehr leicht löst, so erhält man aus Dextrose etwa $50 \%$, aus Lävulose über $100 \%$ Rohosazon. N e u berg schreibt vor, die Mischungen einige Minuten im siedenden Wasserbade oder 24 Stunden auf $40^{\circ} \mathrm{zu}$ erwärmen; in diesem Falle verläuft die Osazonbildung zwar schneller, aber viel weniger glatt; man erhält zunächst auch aus Lävulose ölige Ausscheidungen, die $\mathrm{N}$ e u b e r g

11) Berl. Berichte 1889,90 . durch starke Abkühlung mit flüssiger Kohlensäure zum Kristallisieren bringen muß. Eine solche Arbeitsweise, welche den glatten Verlauf einer Reaktion verhindert, ist nicht zu empfehlen, sie kann leicht zu Täuschungen führen, zumal unter den Bedingungen $\mathrm{N}$ e u bergs auch aus reiner Dextrose immer etwas Methylphenylosazon in Kristallen isoliert werden kann.

Zum Nachweis der Lävulose hat somit das Methylphenylhydrazin nur beschränkten Wert. Entsteht das Osazon in der Kälte schon binnen 20 Stunden reichlich, so ist die Anwesenheit von Lävulose ziemlich sicher erwiesen; lävulosearme Gemische aber und solche, welche viel Nichtzucker enthalten, reagieren langsamer und pflegen auch in der Kälte zunächst nur ölige Produkte abzuscheiden. Immerhin waren mit meinen obengenannten Sirupen $\mathrm{P}_{4,5}$ und $\mathrm{R}_{2,3}$ rasch Kristallisationen von Methylphenylosazon zu erzielen.

Von den Eigenschaften des Methylphenylosazons hebe ich noch folgende hervor. Nach Entfernung des Harzes kristallisiert es am besten aus heißem 25\%igem Alkohol oder aus heißem Benzol in langen orangefarbenen Nadeln von ziemlich scharfem Schmelzpunkt $155-158^{\circ}$; es ist in Wasser fast unlöslich, in kaltem absolutem Alkohol bis zu etwa $0,7 \%$ löslich. In dieser absolutalkoholischen Lösung ist es im Natrium. licht bei $40 \mathrm{~cm}$ Rohrlänge polarisierbar und besitzt ein spezifisches Drehungsvermögen von etwa $[\alpha]_{\mathrm{D}}+48^{\circ}$. Die Körper aus Lävulose und Dextrose sind völlig identisch. Durch Eindampfen seiner wässerigen oder alkoholischen Lösungen oder durch Erhitzen über $110^{\circ}$ wird es zersetzt.

Als entscheidender Nachweis der Lävulose kann somit nur die Fällung des Calciumlävulosats bei $0^{\circ}$ und die starke Linksdrehung des daraus abgeschiedenen Zuckers gelten; dieser Fällung muß eine Anreicherung durch Fraktionieren mit absolutem Alkohol und Alkoholäther voraufgehen. Als Bestätigung können die Reaktion nach $\mathrm{S}$ i e b e $\mathrm{n}$ und die Utberführung in Methylphenylosazon dienen.

In einer Reihe schöner Arbeiten haben de B r u y n und van E kenste in nachgewiesen, daß Dextrose in schwach alkalischen Lösungen teilweise in Lävulose und Mannose umgelagert wird, und weiter, daß diese Umlagerung umkehrbar ist ${ }^{12}$ ). Meine kleine Studie liefert den Beweis, daß Dextrose auch mit Säuren teilweise in Lävulose übergeht; in diesem Falle war aber Mannose

12) v on Lippmann, S. 342 . 
nur in Spuren nachzuweisen, und ob der Vorgang umkehrbar ist, muß durch weitere Versuche geprüft werden. Wahrscheinlich spielt diese Umlagerung im Pflanzenreiche eine Rolle; die Zu- und Abnahme der Dextrose bzw. Lävulose in den Fruchtsäften wird nicht bloß durch Enzyme, sondern auch durch den Säuregehalt der Säfte beeinflußt werden.

\section{Fortschritte auf dem Gebiete der ätherischen Öle und Riechstoffe im Jahre I904. \\ Von F. Rochussen. (Schlufs von S. 1134.)}

Entsprechend der Bedeutung der Aldehyde für die Riechstofftechnik sind die synthetischen Methoden zu ihrer Gewinnung im Berichtsjahr wesentlich erweitert worden; eine Reihe von Verfahren, die auf technische Verwendung Anspruch erheben, ist oben kurz erwähnt worden. Neuerdings benutzt man als Ausgangsmaterial Derivate der Ameisensäure, wieOrthoameisenester(Tschits c h i b a b i n ${ }^{51}$ ), B od r o u x ${ }^{52}$ )), Ameisenester (Gattermann und Maf -

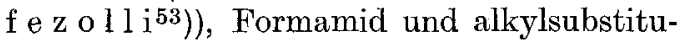
ierte Formamide (Bé h a l und So m me le t ${ }^{54}$ ) die mit Grignardschen Organomagnesiumverbindungen in Reaktion gebracht werden. D a r z e n s ${ }^{55}$ ) kondensierte $\mathrm{Ke}$ tone mit $\alpha$-Halogenfettsäureestern und gelangte nach Spaltung der entstandenen $a$-Oxyakrylsäureester zu Aldehyden der Formel

$$
\underbrace{\mathrm{R}}_{\text {Ketonrest }} \underbrace{\mathrm{R}_{\mathbf{1}}}_{\text {Fettsäurerest }}
$$

$\mathrm{B} l \mathrm{l}$ is $\mathrm{e}^{56}$ ) ging von den Laktiden von $\alpha$-Oxysäuren aus, die bei der trockenen Destillation in Kohlenoxyd und Aldehyd zerfallen sollen nach dem Schema:

$$
\begin{aligned}
& 2 \mathrm{R} \cdot \mathrm{CHOH} \cdot \mathrm{COOH} \rightarrow \mathrm{O} \backslash \stackrel{\mathrm{CH}-\mathrm{CO}-\mathrm{CH}}{\mathrm{R}} \mathrm{O} \\
& \rightarrow 2 \mathrm{R} \cdot \mathrm{CHO}+2 \mathrm{CO} \text {. }
\end{aligned}
$$

Vom Piperonal (Heliotropin) ausgehend, stellte Mameli ${ }^{57}$ ) durch Behandlung

51) Berl. Berichte 37, 286 (1904).

52) Compt. r. d. Acad. d. sciences 138, 92, 700 (1904)

53) Berl. Berichte 36, 4152 (1903).

54) Compt. r. d. Acad. d. sciences 138, 89 (1904). (1904).

55) Compt. r. d. Acad. d. sciences 139, 1214 (1904).

56) Compt. r. d. Acad. d. sciences 138, 697

57) Atti R. Acc. d. Linc. (5) 13, II, 315 (1904). - Nach Chem. Centralbl. 1904, II, 1567. mit Alkylmagnesiumhaloiden sekundäre Alkohole der allgemeinen Formel $\mathrm{CH}_{2} \mathrm{O}_{2} \cdot \mathrm{C}_{6} \mathrm{H}_{3}$ . $\mathrm{CH}(\mathrm{OH}) . \mathrm{R}$ dar, und $\mathrm{E} r \mathrm{l}$ e $\mathrm{n} \mathrm{m}$ e $\mathrm{y}$ e $\mathrm{r}$ und B ra un ${ }^{58}$, kondensierten Piperonal bzw. $\mathrm{Z}$ i m t a l d e h y d mit Phenylbrenztraubensäure zu $\mathrm{Oxolaktonen}$

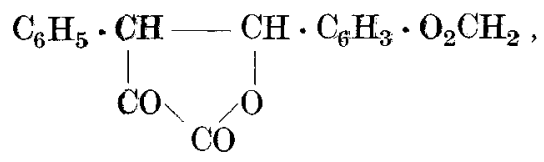

ausgezeichnet durch ihre Umsetzungsfähigkeit.

Nach L e r a t ${ }^{59}$ ) geht $V$ a $n$ ill i n durch die Oxydasen des Preßsaftes verschiedener Pilze der Gattung Russula (Champignons), auch durch Lösungen von arabischem Gummi, in Dehydrodivanillin über; ähnlich wie früher von anderer Seite unter denselben Bedingungen der Übergang von Morphin in Dehydrodimorphin beobachtet wurde.

Die Halogenderivate des $\mathrm{K}$ a $\mathrm{m}$ p fers und ihre Umwandlungen behandelte $B r \ddot{u} \mathrm{~h} \mathrm{I}^{60}$ ) in mehreren Veröffentlichungen.

Die Anwendung des Natriumamids zur Kondensation von Citral und Aceton (Darstellung von $\psi$-Jonon) ist oben kurz erwähnt worden. Das $\mathrm{NaNH}_{2}$ hat den wesentlichen Vorteil gegenüber dem Natrium, daß Wasserstoffbildung und somit auch eine teilweise Reduktion der Ausgangsmaterialien vermieden wird. Haller ${ }^{61}$ ) und seine Schüler bedienten sich des Natriumamids zur Synthese von alkylsubstituierten $\mathrm{M}$ e $\mathrm{n} \mathrm{t} h$ o $\mathrm{n}$ e $\mathrm{n}$ aus Menthon und Halogenalkylen; bei den höheren Homologen dieser Körperklasse wurden eigentümliche Anomalien in bezug auf Drehung und Siedepunkt wahrgenommen.

Die aus verschiedenen ätherischen ölen isolierten $\mathrm{Thujone}$ untersuchte $\mathrm{Wal}$ 1 a $\mathrm{ch}$ 62) zur Entscheidung der Erage, ob dieselben physikalisch identisch oder nur isomer seien. Die Nachprüfung ergab das letztere. Mit Sicherheit gelang. der Nachweis zweier Thujone, die als $\alpha$ - und $\beta$-Verbindung unterschieden werden. Ersteres ist linksdrehend und liefert zwei rechtsdrehende Semicarbazone vom F. 186-188 und $110^{\circ}$. Es kommt als Hauptbestandteil im Thujon des Thujä̈ls vor, ferner im Artemisia- und Salbeiöl. Das $\beta$-Thujon ist rechtsdrehend, jedoch kein Antipode des $\alpha$-Isomeren, und kann in ein hexagonales, ebenfalls rechts drehendes Semicarbazon vom F. $174-176^{\circ}$ übergeführt werden, das leicht

58) L i e big s Ann. 333, 254 (1904).

59) J. pharm. et chim. (6) 19, 10 (1904).

60) Berl. Berichte 37, 2156, 2163, 2178 (1904).

61) Compt. r. d. Acad. d. sciences 138, 1139 (1904).

62) L i e bigs Ann. 336, 247 (1904). 